\title{
Shale gas permeability upscaling from the pore-scale ${ }^{a)}$
}

Lefki Germanou, ${ }^{1,2, b)}$ Minh Tuan Ho, ${ }^{1,3}$ Yonghao Zhang, ${ }^{1,3}$ and Lei $\mathrm{Wu}^{1,4}$

1) James Weir Fluids Laboratory, Department of Mechanical and

Aerospace Engineering, University of Strathclyde, Glasgow G1 1XJ,

$U K$

2) Current Address: TÜV SÜD National Engineering Laboratory,

Scottish Enterprise Technology Park, East Kilbride, G75 OQF, $U K$

${ }^{3)}$ Current Address: School of Engineering, University of Edinburgh, Edinburgh EH9 3FB, UK

4) Current Address: Department of Mechanics and Aerospace Engineering, Southern University of Science and Technology, Shenzhen 518055, China

(Dated: 18 September 2020) 
The effective permeability of large shale samples provides useful insights for shale gas production. However, its determination can only be achieved through upscaling, since the direct pore-scale simulation of gas flows in large rock samples is not feasible due to the high computational cost and absence of pore connectivity in sample images. Although the Brinkman formulation is widely used in the permeability upscaling of conventional rocks, how to choose the effective viscosity in this coarse-scale model is not clear. Moreover, its application in shale rocks, where the rarefaction effects are important so that the conventional Navier-Stokes equations are inadequate, is rare and its accuracy has not been assessed. This study aims to address the above two problems, by comparing the Brinkman solutions of several two- and three-dimensional random porous media containing fractures with the fine-scale solutions of the Stokes and Boltzmann equations (for continuum and rarefied gas flows respectively). It is found that the use of the fluid viscosity on the Brinkman model, instead of the controversial effective viscosity, leads to accurate results for the cases considered. Additionally, the macroscopic quantity in rarefied gas flows in shale rocks is well predicted by the Brinkman model for a wide range of gas rarefaction, since the error is found to be less than $7 \%$, while neglecting the rarefaction effects leads to significant underestimation of effective permeability (up to $90 \%$ in the cases studied). Although heterogeneity and anisotropy of the porous medium increase the error of the effective permeability derived from the Brinkman model, generally speaking, the effective permeability extracted from this coarse-scale model compares favourably to its finescale counterpart.

Keywords: Brinkman equation, upscaling, porous media, shale gas permeability, rarefied gas flow

\footnotetext{
a) Dedicated to the memory of Professor Jason Reese.

b)Electronic mail: lefki.germanou@gmail.com
} 


\section{INTRODUCTION}

Shale formations are characterised by heterogeneity across multiple scales, which makes the accurate determination of their effective properties, in particular permeability, a challenging task. In the presence of this structural heterogeneity effective permeability depends not only on rarefaction effects (such as the velocity slip at the solid surface), but also on scale. Typically, the shale matrix is composed of pores with size less than $2 \mathrm{~nm}$ up to more than $200 \mathrm{~nm}$, and the majority of the pores measured are micro- and mesopores thus the average pore size is less than $20 \mathrm{~nm} \cdot{ }^{1,2}$ In this case, the shale matrix is characterised by low-speed rarefied gas flow, mainly in the slip and transition regimes. ${ }^{3}$ The gas kinetic equation $^{4-7}$ should be used to describe the rarefied gas flow, and the apparent permeability will be much larger than the intrinsic permeability. Shale rocks also contain microfractures with the size much larger than the micro- and mesopores ${ }^{8,9}$, hence the gas flow through microfracture can be described by the Navier-Stokes equations.

The multi-scale nature of shale rock and imaging techniques imposes the necessity of employing upscaling strategies for the calculation of permeability. For example, the current high-resolution images provide a pore space which connects across the sample, making the direct numerical simulation feasible. Nevertheless, their size $(S)$ is limited to a few microns. ${ }^{1}$ Moving to larger samples and lower resolutions $(S>100 \mu \mathrm{m})$ the connectivity of the pores is not detectable while the microfractures are visible; therefore upscaling is the key to communicating data between different scales.

One of the hybrid models that allow the solution of the gas flow in a computational domain containing both porous and free flow regions is the Brinkman approach. ${ }^{10}$ However, to the best of our knowledge, a detailed study comparing the fine-scale effective permeability of ultra-tight porous media with fractures against permeability predicted by the coarse-scale Brinkman model has not been performed yet. So far most of the published works assume a priori that the use of this approach is associated with little or no error. Soulaine et al. ${ }^{11}$ used the Brinkman approach to resolve the flow for shale images that include some voxels with pores that lie below the instrument resolution. The focus of their research is on the impact of this sub-resolution porosity on permeability. Keller and Holzer ${ }^{12}$ upscaled permeability from the pore-scale using multi-scale imaging data, where the Brinkman equation was applied to predict the mesoscale permeability of two samples, which was then favourably compared 
to the corresponding experimental values. The Brinkman framework has also been extensively used in other publications. ${ }^{13,14}$ However, the effectiveness of the Brinkman equation in permeability upscaling for shale still requires a thorough investigation, especially when the rarefied gas flow is present in shale rocks.

To this end, we perform an extensive study of the gas flow in two-dimensional (2D) and three-dimensional (3D) random porous media containing a fracture, where the Brinkman estimation of the effective permeability is assessed against the permeability obtained from the direct numerical simulation on the exact structure, with the aim to investigate the suitability of the Brinkman equation in the upscaling of shale permeability from the pore-scale. Herein, we evaluate for the first time the use of this model in rarefied gas flows.

The rest of this paper is constructed as follows. In Sect. II, the gas flow problem is analysed and the method to calculate permeability is described. Additionally, governing equations and numerical schemes are presented along with the geometry generation method. In Sect. III A, the impact of various morphological characteristics such as porosity, pore size, anisotropy, fracture aperture, on the accuracy of Brinkman model for gas flows in the continuum regime is assessed in 2D structures. Moreover, various 3D porous media generated to mimic real pore-scale shale samples are tested, which provides useful insights pertaining to the application of the Brinkman approach for permeability upscaling. In Sect. III B, the deviation of the macroscopic model for permeability upscaling in multiscale porous structures (i.e. the gas flow is rarefied at the finest scale) is examined across a wide range of rarefaction parameter. Finally, the paper concludes in Sect. IV.

\section{GOVERNING EQUATIONS AND SIMULATION SETUP}

\section{A. The flow problem and upscaling method}

The problem of momentum transport along the boundary between a fluid layer and a porous medium has been extensively studied over the past decades. The motivation behind this lies in the variety of applications where this configuration can be found, such as ground water pollution, catalytic and nuclear reactors, oil and gas recovery among many others.

Beavers and Joseph ${ }^{15}$ conducted experiments of a 2D Poiseuille flow through a rectangular channel bounded by an impermeable upper wall and a permeable lower wall. The Stokes 
equation, which is satisfied in the so-called free region, and the Darcy's law, which is satisfied in the bulk of the porous region, are coupled using the following well-known semi-empirical slip boundary condition at the interface (dividing surface between the two distinct regions ${ }^{16}$ ):

$$
\left.\frac{\mathrm{d} \bar{u}}{\mathrm{~d} y}\right|_{y=0^{-}}=\frac{\alpha}{\sqrt{k}}\left(\left.\bar{u}\right|_{y=0}-\bar{u}_{D}\right)
$$

where $\alpha$ is the slip coefficient, $\bar{u}$ is the volume averaged velocity, $\bar{u}_{D}$ is the Darcy velocity (i.e. the volume averaged velocity in the bulk of the porous medium), and $k$ is the isotropic permeability of the porous medium. The relationship between $\bar{u}_{D}$ and $k$ is determined by the Darcy law, i.e.

$$
\bar{u}_{D}=-\frac{k}{\mu} \frac{\mathrm{d} p}{\mathrm{~d} x}
$$

where $\mathrm{d} p / \mathrm{d} x$ is the imposed pressure gradient, and $\mu$ is the fluid viscosity. The validity of the Darcy equation is extensively proven for low-Reynolds number porous media flows.

The Beavers and Joseph boundary condition (1) was derived to account for the fact that, according to the experimental results, the interfacial velocity $\left.\bar{u}\right|_{y=0}$ is significantly greater than the Darcy velocity $\bar{u}_{D}$. This indicates the presence of a thin transition zone that forms at the porous region near the interface, where a gradual enhancement of the viscous shear is taking place. The slip coefficient $\alpha$ is an adjustable parameter that depends on the local structure of the transition zone and the flow properties. It should be distinguished from the one in rarefied gas dynamics, ${ }^{17}$ which is due to the rarefaction effect (i.e. infrequent collisions between gas molecules to fully thermodynamically equilibrate the gas).

The use of Eq. (1) that leads to a discontinuity of the velocity at the interface can be circumvented by the implementation of other formulations such as the widely spread model of Brinkman. ${ }^{10}$ In this approach, the continuity of both velocity and stress is ensured, coupling the momentum equations that govern the free fluid and porous regions (Stokes and Darcy respectively). This hybrid model can be regarded as an extension to Darcy's law, as it includes a macroscopic shear term that accounts for the velocity gradient present at the transition zone and the free region. The Brinkman momentum equation which applies to the whole domain reads

$$
0=-\nabla p+\mu_{e f f} \nabla^{2} \overline{\mathbf{u}}-\frac{\mu}{\mathbf{k}} \overline{\mathbf{u}}
$$

where $\overline{\mathbf{u}}=\left(\bar{u}_{x}, \bar{u}_{y}, \bar{u}_{z}\right)$ is the vector of the volume averaged velocity, $\mathbf{k}$ is the permeability 
tensor, and $\mu_{e f f}$ is the so-called effective viscosity that is presumed constant in Brinkman's original model. The last term in the right-hand side of Eq. (3) is a drag term which is meaningful and dominant only if the control volume is a porous medium; otherwise, this term vanishes as $k \rightarrow \infty$. In the former case, the momentum equation can be approximated by the Darcy law (with the exception of transition zone), while in the latter it reduces to the Stokes equation. It should be emphasised, that if $\mu_{e f f} \neq \mu$, the effective viscosity should not be considered as the real viscosity of the fluid in the porous medium. Nevertheless, in the homogeneous fluid region the flow field should be consistent with the Stokes equation, thus the original fluid viscosity, $\mu$, must be applied there.

Even though the widely known Brinkman equation has been the focus of many researchers over the last decades, general agreement has not been reached yet regarding the definition of effective viscosity. Effective viscosity was found, to be either smaller, equal or greater than the fluid viscosity, often following heuristic approaches. ${ }^{18,19}$ Specifically, according to the Stokes simulations performed in Martys et al. ${ }^{20}$ and their comparison with the respective Brinkman simulations, it was found that $\mu_{e f f} / \mu$ exceeds unity. In order to match the two simulation results, effective viscosity should increase as the porosity, $\epsilon$, of the medium decreases. Volume averaging performed by Ochoa-Tapia and Whitaker ${ }^{21}$ resulted in $\mu_{\text {eff }}=$ $\mu / \epsilon$. Valdes-Parada et al. proposed a more complex form of the effective viscosity. ${ }^{22}$ Sahraoui and Kaviany ${ }^{23}$ used a periodic porous medium made of in-line or staggered arrangements of cylinders to study the flow effects at the vicinity of the free flow region. In their study, it was concluded that the effective viscosity should vary within the porous medium so that the Brinkman model can provide satisfactory prediction of the velocity near the interface. Although this is a controversial subject, it seems to be generally accepted that effective viscosity depends on the nature of porous medium and the flow. However, the conditions of applicability of the model are also questioned in the literature, ${ }^{18}$ thus its validation for the cases of interest is essential. Here we must remark that the assumption $\mu_{e f f} / \mu=1$ is retained in this study, as in the original paper of Brinkman, ${ }^{10}$ owing to the lack of a definitive conclusion for this ratio from the research community.

The Brinkman formulation is used herein to simulate the flow in fractured porous media. The matrix is described by free region with effective properties (permeability tensor), therefore the explicit representation of its structure is avoided and the flow is governed by Darcy's law. Conversely, the fractures are considered 'open' channels (having infinite permeability) 
and thus the gas dynamics are governed by the Stokes equation. The flow is assumed to be isothermal and incompressible and the Reynolds number is less than one.

\section{B. Geometry generation}

The $2 D$ and $3 D$ porous media structures used in this work are generated by the Quartet Structure Generation Set (QSGS) method. ${ }^{24}$ The use of this algorithm for the generation and study of artificial shale samples is common in the literature. ${ }^{25-28}$ This process reconstructs random porous media using three controlling parameters, $P, c_{d}$ and $D_{i}$, which indirectly determine the porosity, the size of the solid elements and the level of anisotropy, respectively. The algorithm is briefly described as follows.

1. Randomly generate cores in the uniform Cartesian grid based on the core distribution probability $c_{d}$.

2. Expand the growing elements according to the given directional growth probability $D_{i}$. For $2 D$ structures we have $1 \leq i \leq 8$, while for $3 D$ we have $1 \leq i \leq 26$, where $i$ is an integer. The main directional growth probabilities, $D_{\text {main }}$, are the ones in alignment with the axis, i.e. $D_{x}, D_{y}, D_{z}$.

3. Repeat the previous step until the volume fraction of solid elements has reached the desired value $P$.

In our analysis, we use the porosity $\epsilon=P-1$ and the aspect ratio $A R$ in addition to the aforementioned parameters. The aspect ratio is defined as the ratio of the maximum to the minimum $D_{\text {main }}$, thus, reflecting the degree of anisotropy.

Heterogeneity cannot be directly controlled using the initial form of the algorithm, however, Wang et al. ${ }^{27}$ recently proposed a two-level method to accomplish it. The algorithm is briefly described as follows.

1. Generate a refined structure with distinct values of core distribution probability $c_{d}^{R}$, directional growth probabilities $D_{i}^{R}$, and volume fraction $P^{R}$.

2. Create another coarse structure using a new core distribution probability $c_{d}^{C}<c_{d}^{R}$ to construct the cores, and then expand them using $D_{i}^{C}$. 
3. The coarse structure grows until the combined result of the two structures reaches the desired volume fraction $P$.

It is noted that heterogeneity is an increasing function of the ratio $c_{d}^{R} / c_{d}^{C}$. It should also be stressed that for the generation of 2D QSGS structures, the growing phase is selected to be the solid as in our previous work, ${ }^{5}$ while for the 3D structures fluid is the growing phase. This choice is based on the numerous examples of real shale sample images. ${ }^{1,27}$ Furthermore, in all the produced geometries the final porosity $\left(\epsilon_{\text {final }}\right)$ is smaller than the nominal one $(\epsilon)$ due to the fact that a portion of non-connected pores were generated. ${ }^{28}$ Finally, it should be noted that in this paper grid refers to the QSGS matrix generated using the above method, while the term mesh refers to the corresponding computational mesh used in numerical simulations.

\section{The determination of permeability tensor}

The calculation of the full permeability tensor $\mathbf{k}$ of a 3D porous medium requires three flow simulations, imposing pressure gradients in the $x, y$ and $z$ directions, respectively. Each simulation for each spatial direction gives one column of the tensor. For example, using Darcy's law (2) and imposing $\nabla p_{x}$ the first column of the permeability tensor is obtained by

$$
k_{i x}=-\frac{\bar{u}_{i} \mu}{\nabla p_{x}}, \text { for } i=x, y, z,
$$

where $\bar{u}_{i}$ is the $i$-th component of the Darcy velocity $\overline{\mathbf{u}}$ that is defined as

$$
\overline{\mathbf{u}}=\frac{1}{V} \int_{V_{f}} \mathbf{u d} V
$$

Here, $V_{f}$ is the volume occupied by the fluid and $V$ the total volume of the selected region. We note that when the porous medium is $2 \mathrm{D}$, the order of the system is reduced to $2 \times 2$. Finally, we should remark that the nature of the permeability tensor depends on the characteristics of the porous medium and the flow configuration such as the boundary conditions. ${ }^{29,30}$ Herein, $\mathbf{k}$ is by default non-symmetrical. 


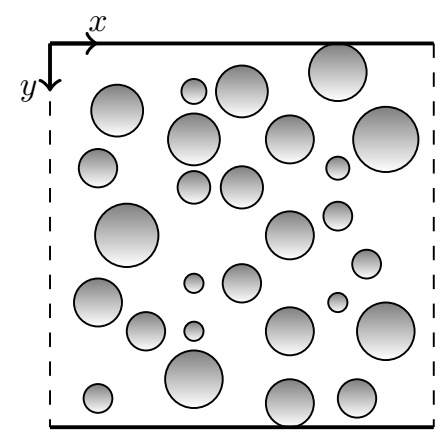

(a) Permeability determination

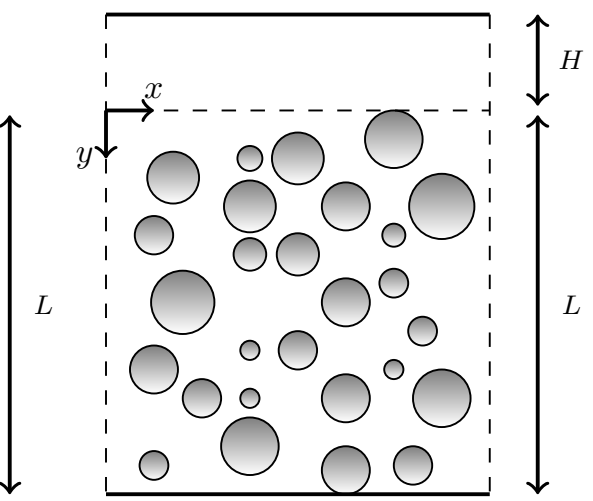

(b) Fine-scale model

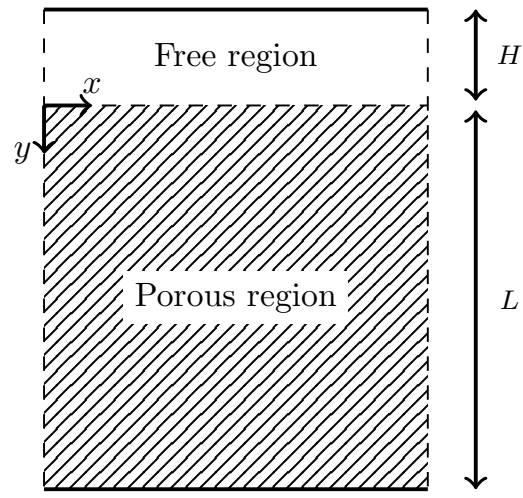

(c) Coarse-scale model

FIG. 1: Schematic of the accuracy assessment of the Brinkman model for permeability upscaling. (a) The permeability tensor $\mathbf{k}$ of the porous medium is calculated. (b) The fine-scale model is simulated to find the effective permeability $k_{\text {eff }}^{F}$ of the system consisting of the porous medium (porous region) of size $L$ and a straight channel (free region) of thickness $H$. The porous structure and the channel share a common interface indicated by the dashed line at $y=0$. (c) The coarse-scale model, where the porous medium is treated as free region but with a permeability tensor $\mathbf{k}$, is simulated using the Brinkman equation.

The obtained effective permeability is denoted as $k_{\text {eff }}^{C}$. Each region is meshed using individual blocks as explained in detail in Table I.

\section{Governing equations and numerical methods}

Numerical simulations performed in this paper are distinguished in three main classes: (a) the Stokes and Boltzmann equations are solved on the explicit representation of the porous matrix in order to extract the intrinsic and apparent permeability tensor, respectively, (b) the Stokes and Boltzmann equations are solved on the explicit representation of the porous matrix and fracture in order to obtain the effective permeability of the fine-scale model $k_{e f f}^{F}$, and (c) the Brinkman equation is solved on the coarse-scale model, where the porous medium is represented through its effective properties, in order to acquire the effective permeability approximation $k_{\text {eff }}^{C}$. For instance, 2D computational domains of the above simulation categories are shown in Fig. 1.

\section{Stokes and Brinkman equations}

In the continuum flow regime, the incompressible single-phase gas flow is described by the Stokes equation in the fine-scale model and by the Brinkman equation in the coarse-scale 
model. These equations are solved in the framework of Semi-Implicit Method for Pressure Linked Equations (SIMPLE) algorithm and all the discretisation schemes are second order. Fixed-pressure boundary conditions are applied at the inlet and outlet of the domain, while all lateral boundaries are treated as solid walls. Alternatively, when stated, the symmetry boundary condition is imposed on lateral walls. The pressure difference is maintained low to assure that the Reynolds number is less than one. This simulation setup is considered to provide correct diagonal and off-diagonal permeability terms. ${ }^{30}$

The pore-scale flows in QSGS structures and their respective macroscopic Darcy-like representations are evaluated using the finite volume method implemented in the opensource CFD toolbox OpenFOAM. The numerical solution of Stokes equation requires the binary matrix generated by the QSGS method $(1000 \times 1000$ grid for $2 \mathrm{D}$ geometry and $100 \times 100 \times 100$ grid for 3D geometry) to be transformed into a computational mesh, of at least the same resolution, consisting of the pore space only. The native OpenFOAM mesher, snappyHexMesh, is utilised to produce a hexahedral mesh for each geometry. For 3D cases, the resolution is doubled in order to increase accuracy.

Most cases studied in this paper follow the configuration shown in Fig. 1. When the channel is added on top of the domain the mesh then consists of two blocks. The porous region is meshed as previously mentioned, however the number of mesh corresponding to the channel is much smaller, by applying mesh refinement towards the interface between the free and porous region, and the top boundary.

On the other hand, the numerical solution of the Brinkman equation does not necessarily require the same mesh resolution. To reduce the computational cost while maintaining accuracy, a coarser hexahedral mesh is constructed for the whole domain, where multigrading is applied to achieve mesh refinement in the areas of interest, i.e. towards the top and bottom boundary and most importantly, in the vicinity of the interface between the free and the porous regions. This is again achieved using two blocks corresponding to the porous and free region, respectively. Generally speaking, refinements around the fractures are necessary to properly solve the steep gradients in the region. The mesh information for both $2 \mathrm{D}$ and $3 \mathrm{D}$ cases where a straight channel of thickness $H=0.05 \mathrm{~L}$ is added along the $y$ axis, on top of the porous domain, is displayed in Table I. 
TABLE I: Mesh resolution for 2D and 3D cases for an indicative fracture of $H=0.05 L$ placed on top of the porous domain. The $3^{\text {rd }}, 4^{\text {th }}$ and $5^{\text {th }}$ columns correspond to the mesh size of the domains illustrated in Fig. 1(a), (b), and (c), respectively.

\begin{tabular}{|c|c|c|c|}
\hline & \multicolumn{2}{|c|}{ Stokes } & Brinkman \\
\hline & \multicolumn{3}{|c|}{ Permeability determination Fine-scale model Coarse-scale model } \\
\hline \multirow{2}{*}{$\begin{array}{l}\text { 2D } \begin{array}{l}\text { porous block } \\
\text { channel block }\end{array} \\
\end{array}$} & $1000 \times 1000$ & $1000 \times 1000$ & $200 \times 200$ \\
\hline & - & $1000 \times 20$ & $200 \times 20$ \\
\hline \multirow{2}{*}{$\begin{array}{l}\text { 3D porous block } \\
\text { channel block }\end{array}$} & $200 \times 200 \times 200$ & $200 \times 200 \times 200$ & $50 \times 50 \times 50$ \\
\hline & - & $200 \times 20 \times 200$ & $50 \times 20 \times 50$ \\
\hline
\end{tabular}

\section{Gas kinetic theory}

The permeability of gas flows through ultra-tight porous media, such as shale, is significantly enhanced compared to the intrinsic permeability. Klinkenberg ${ }^{31}$ first observed that the so-called apparent permeability increases with the reciprocal of mean gas pressure $1 / \bar{p}$. Since the variation of apparent permeability is linked to rarefaction effects, gas kinetic theory is adopted to describe flow in low permeability porous media. In this paper, the linearised BGK equation (which can be viewed as the relaxation-time approximation of the Boltzmann equation) is solved to obtain apparent permeability. For further details on the method of solution the reader is referred to our previous work. ${ }^{4-7}$

The Knudsen number $K n$ is a key dimensionless parameter which indicates the degree of flow rarefaction and is defined as a ratio of the mean free path of gas molecules $\lambda$ to the characteristic flow length $L$, i.e.:

$$
K n=\frac{\lambda}{L}, \text { and } \lambda=\frac{\mu\left(T_{0}\right)}{\bar{p}} \sqrt{\frac{\pi R T_{0}}{2}}
$$

where $\mu\left(T_{0}\right)$ is the shear viscosity of the gas at a reference temperature $T_{0}, \bar{p}$ is gas pressure, and $R$ is the gas constant. When the temperature is fixed, the Knudsen number is then proportional to $1 / \bar{p}$, so the apparent permeability increases with $1 / \bar{p}$.

Gas flow has been classified into four regimes based on the level of rarefaction: continuum flow $(K n \leq 0.001)$ in which the Navier-Stokes equations are valid, slip flow $(0.001<K n \leq$ 0.1) where the Navier-Stokes equations are still applicable when coupled with appropriate velocity-slip boundary conditions, transition $(0.1<K n \leq 10)$ and free molecular flows 
$(K n>10)$ where the continuum assumption breaks down and gas kinetic equations are needed to describe the rarefied gas flow. It is important to keep in mind that the limits of $K n$ in this classification may vary according to the problem geometry.

The boundary conditions for flow velocities are periodic in the direction of the pressure gradient and symmetry on lateral walls. In order to realize this periodic boundary conditions, the grid in Sect. III B is extended by 5 fluid layers at the inlet and outlet of the domain. This does not affect the permeability calculation, as long as the mean free path of gas molecules is much smaller than the size of computational domain.

\section{RESULTS AND DISCUSSION}

In the simulation results that follow most of the parameters reported are dimensionless, such as the Knudsen number $K n$, and the parameters related to the properties of the QSGS structures. Nevertheless, we note that $H, L, u$ and $p$ are used in their dimensional form.

\section{A. Upscaling continuum flows}

In our numerical computations, we mimic the experimental setup of Beavers and Joseph ${ }^{15}$ using random 2D and 3D porous media generated with the QSGS algorithm. The domain consists of a rectangular channel of variable width $H$ which is placed over a porous block of fixed size $L$, as demonstrated in Fig. 1(b). In our study, several random porous media are utilised in order to showcase the level of accuracy of the Brinkman approach depending on the properties of permeable materials.

In particular, we carry out independent calculations to obtain the permeability tensor $\mathbf{k}$ of selected porous medium as explained in detail in Sect. II C. We then use this $\mathbf{k}$ as input for the Brinkman model where we carry a series of simulations varying the width of the channel, the boundary conditions, and the orientation of the porous medium. The obtained permeability is $k_{\text {eff }}^{C}$. In parallel, we perform simulations of the same configuration using the exact geometry of the matrix. In this way, the fine-scale model, i.e. pore-scale permeabilities $k_{\text {eff }}^{F}$ are obtained through direct simulations and the accuracy of the Brinkman model is then evaluated. The permeabilities $\left(k\right.$ and $\left.k_{e f f}\right)$ mentioned in the rest of this paper are normalised 
TABLE II: Accuracy assessment of the Brinkman model in isotropic QSGS structures with $H=0.05 L$. First three columns: variation of porosity $\epsilon$ while $c_{d}=0.001$. Last three columns: variation of $c_{d}$ while $\epsilon=0.7$. Note that $c_{d}$ controls the size of solid islands, where the larger value corresponds to smaller island.

\begin{tabular}{ccc|ccc}
\hline \multicolumn{3}{c|}{$c_{d}=0.001$} & \multicolumn{3}{c}{$\epsilon=0.7$} \\
\hline$\epsilon$ & $H / d_{\text {mean }}$ & $\eta$ & $c_{d}$ & $H / d_{\text {mean }}$ & $\eta$ \\
\hline 0.6 & 0.94 & $23.08 \%$ & 0.001 & 1.39 & $14.16 \%$ \\
0.7 & 1.39 & $14.16 \%$ & 0.005 & 2.68 & $9.92 \%$ \\
0.8 & 2.13 & $9.12 \%$ & 0.01 & 3.44 & $7.46 \%$ \\
\hline
\end{tabular}

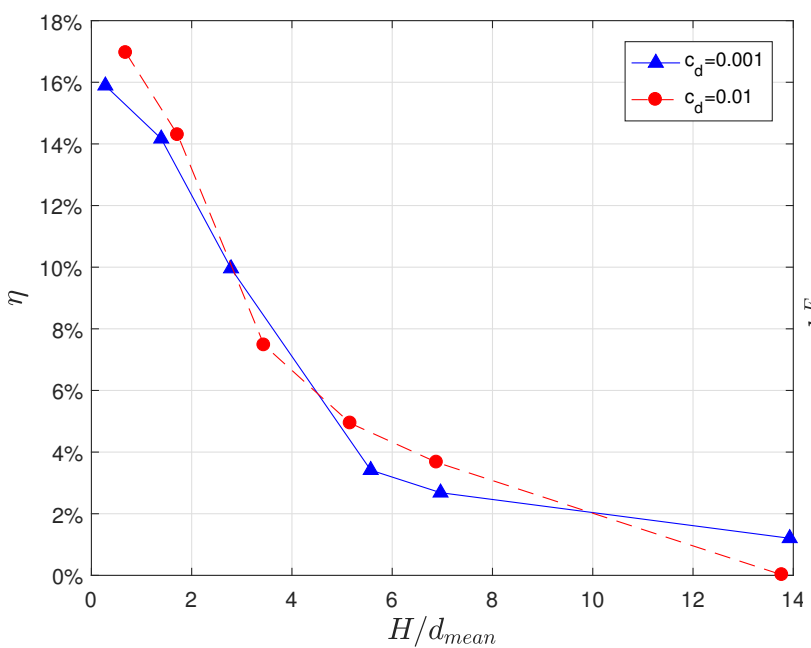

(a)

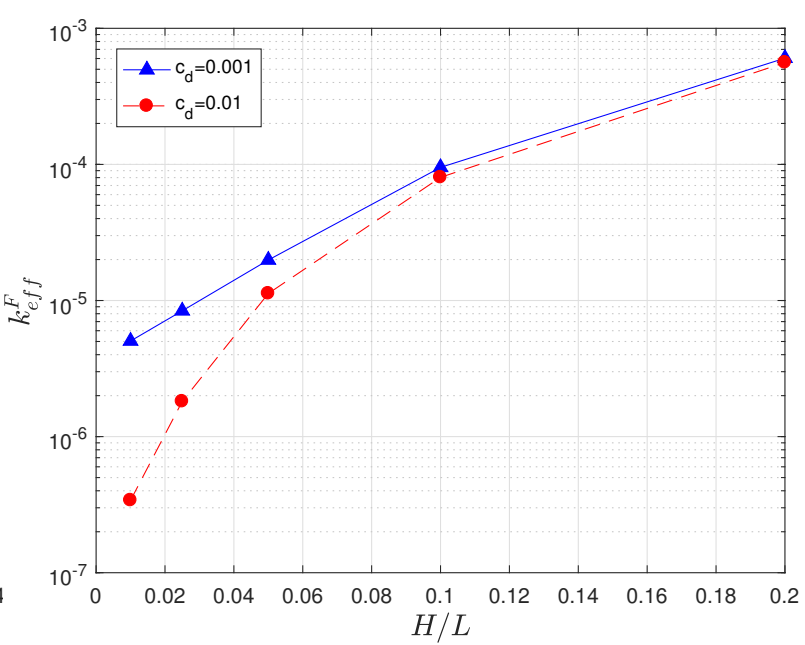

(b)

FIG. 2: (a) Accuracy assessment of the Brinkman model in isotropic QSGS structures and (b) effective permeability evolution when the channel height $H$ varies for the same structures. The porosity is $\epsilon=0.7$, and $c_{d}=0.001$ and 0.01 .

by $L^{2}$ and refer to the diagonal permeability element of the streamwise direction, unless otherwise stated. The relative error of the effective permeability estimation is defined as

$$
\eta=\frac{\left|k_{e f f}^{F}-k_{e f f}^{C}\right|}{\left|k_{e f f}^{F}\right|} 100 \%
$$

\section{Two-dimensional QSGS}




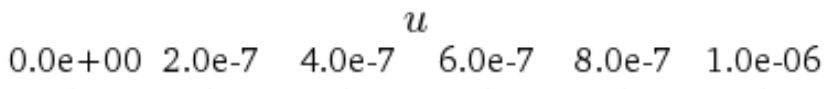

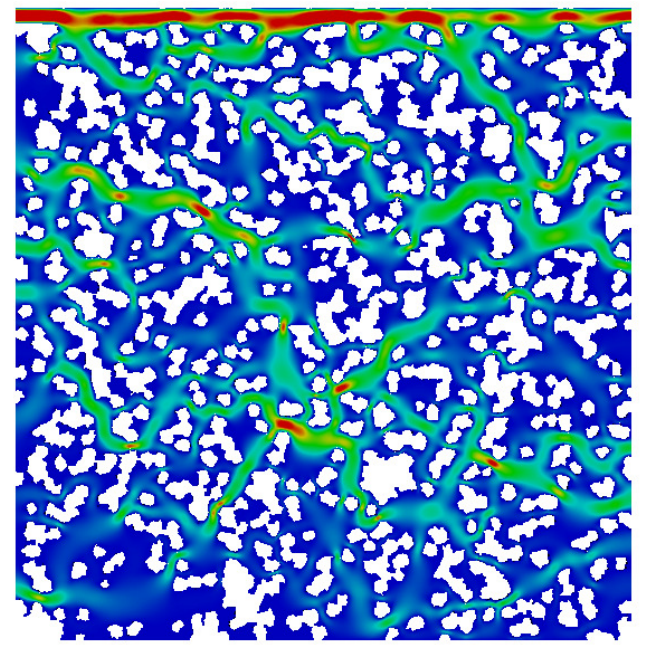

(a)

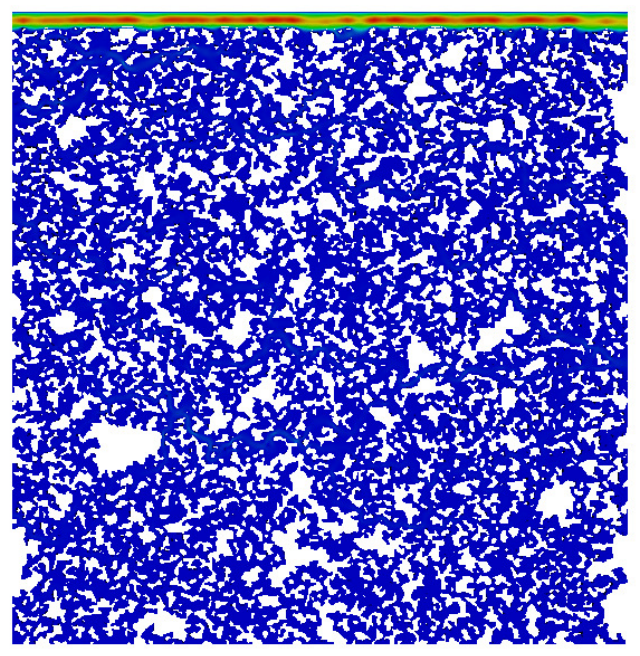

(b)

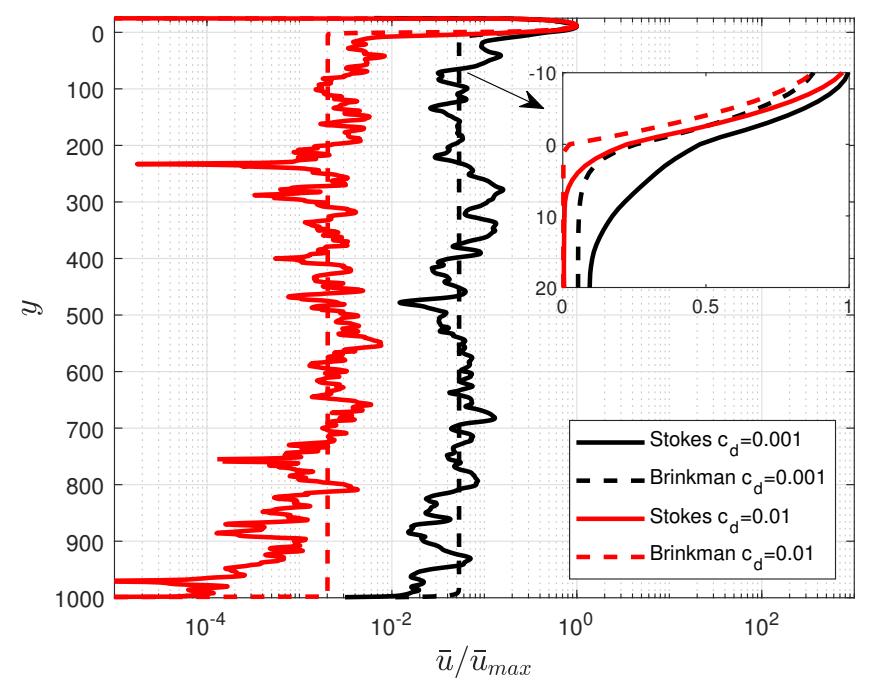

(c)

FIG. 3: Contour plots of the velocity magnitude for the isotropic cases of the same porosity $\epsilon=0.7$ with $c_{d}=0.001$ (a) and 0.01 (b), when $H=0.025 L$. (c) Profiles of the normalised streamwise-averaged velocity for the porous structures shown in (a) and (b), respectively. The transition zone (interface is at $y=0$ ) is smaller for porous media with larger $c_{d}$ since its size is related to the effective pore size.

\section{Isotropic porous media}


To begin with, we investigate the statistically isotropic QSGS geometries $(A R=1)$. Several typical 2D QSGS structures are shown in our previous work ${ }^{5}$. In this context, we maintain the same channel width in all simulations $(H=0.05 L)$. The impact of porosity $\epsilon$, with $c_{d}=0.001$, and effective particle size $c_{d}$, with $\epsilon=0.7$, on the accuracy of the Brinkman equation is shown in Table II. We observe that the relative error monotonically decreases while $\epsilon$ and $c_{d}$ increase, even though these two parameters have an opposite effect on the permeability as analysed in our previous work. ${ }^{5}$ Nevertheless, the common attribute for both the aforementioned parameters is that their enhancement leads to smaller values of the $d_{\text {mean }}$, i.e. the mean diameter of the particles if those are considered to be circular discs.

Recently, Zhang and Prosperetti ${ }^{32}$ remarked that a small ratio of the free region width to the radius of the solids could conceal the physical picture. Therefore, noticing the importance of the $H$ to $d_{\text {mean }}$ ratio we further examine its influence on our cases of interest. We test the isotropic porous structures of $\epsilon=0.7$ and $c_{d}=0.001,0.01$, but vary the channel thickness. Here we must remark that the permeability of these two random porous media used in this comparison differs by 30 times. In agreement with the previous results, Fig. 2(a) shows that the deviation between the permeability calculated from the solution of Stokes and the Brinkman equations drops when $H / d_{\text {mean }}$ increases. The relative error decays to zero in a monotonic fashion and is mostly depending on the value of $H / d_{\text {mean }}$, irrespective of the properties of porous media.

Increasing the channel thickness for the same porous medium, practically decreases the amount (proportion) of fluid passing through the pores of the porous block. In other words, only the channel flow is significant and thus the drag term becomes negligible. Therefore, the Brinkman formulation approaches the Stokes equation, which justifies the small relative error in Fig. 2(a). Moreover, as $H$ grows, the effective permeabilities computed using the two porous structures differ less and less and approach the permeability of the flow in the channel only, see Fig. 2(b).

Decreasing $d_{\text {mean }}$ leads to a more homogeneous porous medium as stated in our previous paper..$^{5}$ It appears that homogeneity favours the Brinkman equation which gives more accurate results as the $H / d_{\text {mean }}$ ratio increases (see Fig. 2). As $H / d_{\text {mean }}$ becomes smaller the relative error reaches its maximum value, which is up to $\sim 23 \%$ for the isotropic cases considered. The simulation results of the two aforementioned isotropic porous media with different particle sizes $\left(c_{d}\right)$, thus different degree of heterogeneity, for the case of $H=0.025 L$ are il- 
lustrated in Fig. 3. Streamwise-averaged velocity profiles are presented here, see Fig. 3(c), since the flow simulations in this paper can be considered quasi-one-dimensional. This is due to the dominance of fractures and due to the small values of the off-diagonal permeability components, compared to the diagonal ones. The velocity contours for the same runs indicate a potential source of error of the Brinkman estimation. We observe from Fig. 3 that in the fine-scale models the shape of the channel is not in fact straight, but due to the existence of some pores adjacent to it, its local thickness is often larger. An additional source of uncertainty is the input permeability tensor, whose value is slightly depending on the flow configuration setup. ${ }^{30}$

\section{Anisotropic porous media}

Moreover, flow simulations using anisotropic QSGS structures are conducted to examine the divergence of permeability from the direct computation. The porous media used herein are generated using the following fixed parameters $\epsilon=0.7, c_{d}=0.001, H=0.05 L$, and for varying values of $A R=100,500,1000$. In our previous paper, ${ }^{5}$ we demonstrated that for such anisotropic porous media without a fracture (see Fig. 1 of that work for similar random porous media), with increasing $A R$ permeability drops when the flow is perpendicular to the bedding plane $(T>>1)$, while it remains in the same order of magnitude for flow parallel to the bedding plane $(T \approx 1)$. Adding a straight fracture on top and comparing the coarsescale with the fine-scale model, the deviation shown in Fig. 4 ranges from approximately $0 \%$ to $24 \%$ for all the tested anisotropic structures.

To reduce the error caused by the randomness of the generation algorithm described in Sect. II B, 5 anisotropic porous structures are created for the same set of generating parameters. Despite the fact that the results are quite scattered, the tendency for the relative error of the Brinkman solution is to increase with anisotropy when the flow is parallel to the bedding plane $\left(k=k_{x x}\right)$ and decrease if otherwise $\left(k=k_{y y}\right)$. In the former case, due to the

orientation of the solid particles (elongated in the horizontal direction), tortuosity $T$ is close to unity while in the latter, where the same porous medium is rotated by $90^{\circ}, T>>1$. At this point we should mention that we calculate tortuosity using the same formulation as in our previous publication, ${ }^{5}$ i.e. $T_{i}=\overline{\mathrm{u}} / \bar{u}_{i}$, where $i$ is the direction parallel to the macroscopic flow. ${ }^{33}$ If not otherwise stated, $T$ herein refers to the tortuosity in the streamwise direction. 


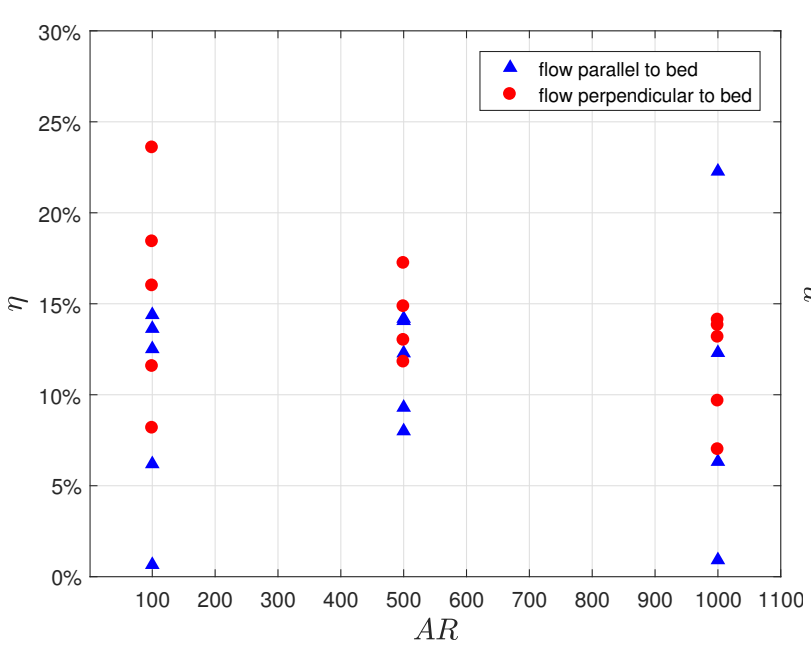

(a)

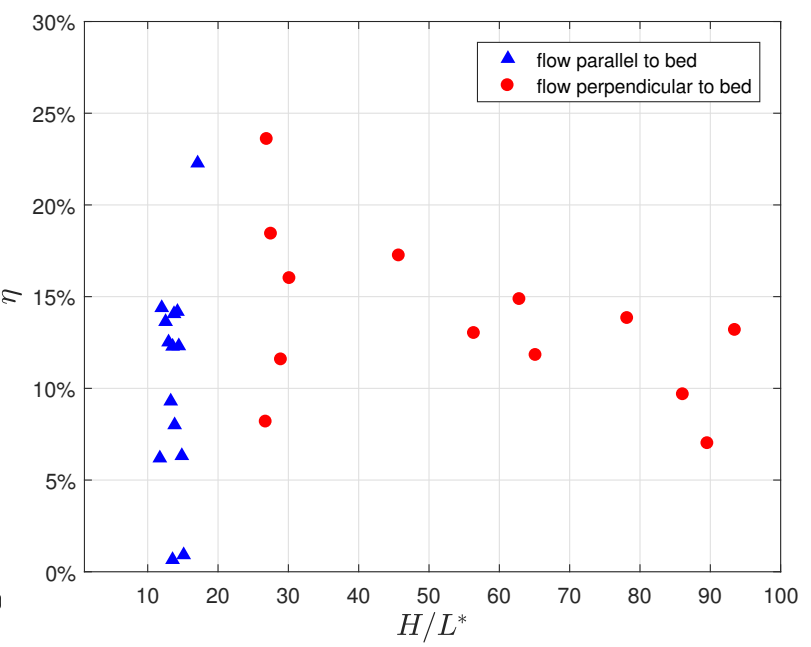

(b)

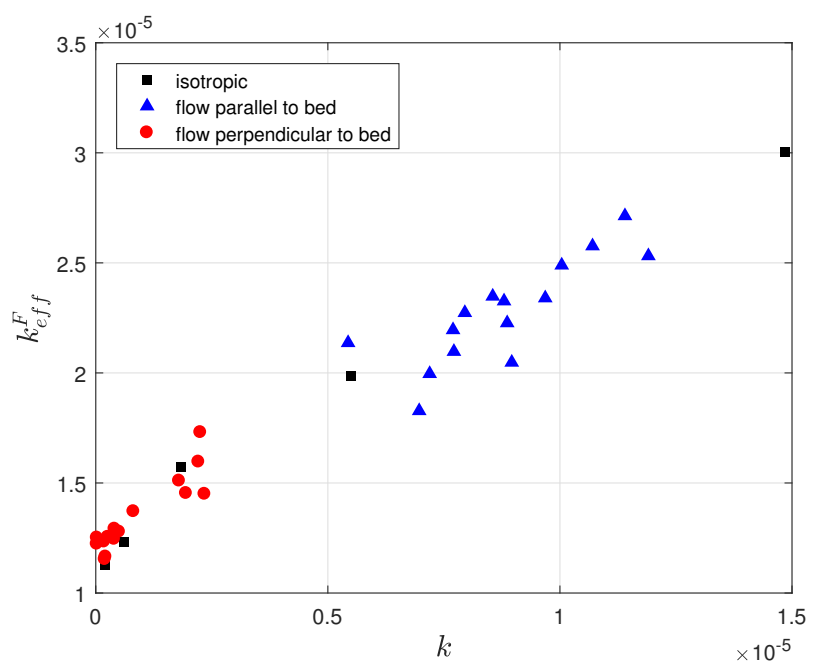

(c)

FIG. 4: (a) and (b) Accuracy assessment of the Brinkman model in anisotropic QSGS structures with $\epsilon=0.7, c_{d}=0.001, H=0.05 L$. (c) The effective permeability of the porous media with one open channel on the top vs. the permeability of the porous media only.

Note that $k=k_{x x}$ if the flow is parallel to the bed and $k=k_{y y}$ if otherwise.

Since the shape of the particles is roughly ellipsoidal in these anisotropic structures, we use the ratio $H / L^{*}$ in the rest of this study. This is calculated utilising the characteristic flow length expression which reads

$$
L^{*}=L \sqrt{\frac{12 k}{\epsilon}} \text { for } 2 \mathrm{D}, L^{*}=L \sqrt{\frac{8 k}{\epsilon}} \text { for } 3 \mathrm{D} \text {. }
$$

Generally speaking, as $A R$ increases, $H / L^{*}$ is almost fixed when the flow is simulated parallel 


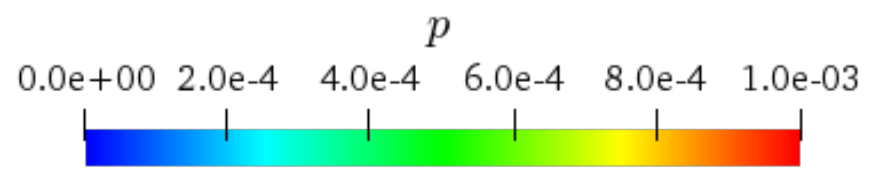

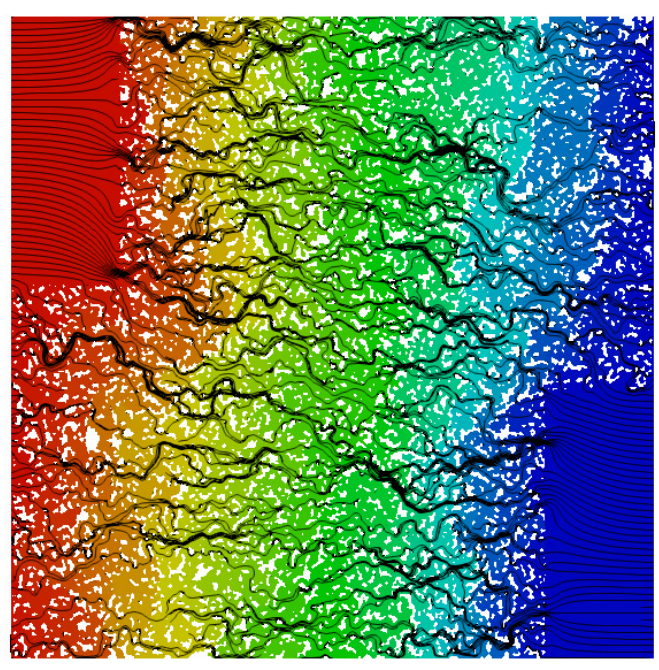

(a)

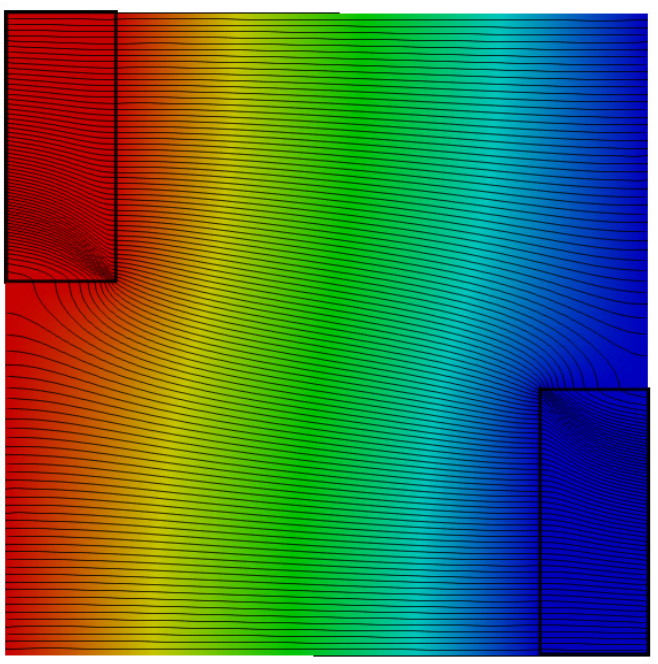

(b)

FIG. 5: Fine and coarse-scale results of the isotropic case generated using $\epsilon=0.7, c_{d}=0.001$, coupled with a fragmented fracture $\left(H=0.4 L, H=173 L^{*}\right)$ along the $x$-axis. Pressure contour plots with velocity streamlines are illustrated for the Stokes (a) and Brinkman solution (b). For clarity, the boundaries of the fracture have been marked with rectangles in (b). The Brinkman model underestimates the effective permeability by $1 \%$.

to bed, in the direction of the elongation of the solid particles $(T \approx 1)$, since $k_{x x}$ remains in the same order of magnitude. ${ }^{5}$ However, when the flow is in the perpendicular direction $(T>>1), H / L^{*}$ increases with $A R$ as $k_{y y}$ drops significantly. A clear correlation for the relative error of permeability with respect to the $H / L^{*}$ cannot be observed for the geometries considered, although it is obvious that it tends to drop for increasing $H / L^{*}$. Consequently, both Fig. 4(a) and (b) yield the conclusion that when the flow is perpendicular to bed, the error associated with the Brinkman model is reduced with increasing anisotropy. Lastly, the plot of the fine-scale effective permeability is an increasing function of the permeability of the porous medium for both isotropic and anisotropic geometries, see Fig. 4(c).

The use of an alternative boundary condition at the upper wall is also investigated. Symmetry boundary condition is applied on the reference configuration of $H=0.05 L$ to 
TABLE III: Generation parameters of 3D QSGS structures and simulation results where $\mathrm{d} p / \mathrm{d} x$ is applied and fracture with $H=0.02 L$ is in $y$ axis. $T$ here refers to the highest component of tortuosity which in the case of anisotropic structures is $T_{z}$.

\begin{tabular}{cccccccccccc}
\hline case & $c_{d}^{R}$ & $c_{d}^{C}$ & $\epsilon^{R}$ & $\epsilon$ & $\epsilon_{\text {final }}$ & $A R$ & $H / L^{*}$ & $k$ & $r$ & $T$ & $\eta$ \\
\hline 1 & 0.01 & - & - & 0.3 & 0.26 & 1 & 5.59 & $4.16 \times 10^{-7}$ & 0.98 & 1.71 & $21 \%$ \\
2 & 0.01 & - & - & 0.3 & 0.26 & 100 & 5.53 & $4.25 \times 10^{-7}$ & 0.73 & 1.68 & $14 \%$ \\
3 & 0.01 & 0.0001 & 0.2 & 0.3 & 0.14 & 1 & 14.73 & $3.27 \times 10^{-8}$ & 0.94 & 2.52 & $21 \%$ \\
4 & 0.01 & 0.0001 & 0.2 & 0.3 & 0.13 & 100 & 16.18 & $2.50 \times 10^{-8}$ & 0.74 & 2.45 & $21 \%$ \\
5 & 0.01 & 0.00002 & 0.2 & 0.3 & 0.13 & 1 & 14.44 & $3.18 \times 10^{-8}$ & 0.77 & 2.36 & $21 \%$ \\
6 & 0.01 & 0.00002 & 0.2 & 0.3 & 0.13 & 100 & 14.30 & $3.22 \times 10^{-8}$ & 0.66 & 2.38 & $21 \%$ \\
7 & 0.01 & 0.0001 & 0.2 & 0.25 & 0.2 & 1 & 9.36 & $1.12 \times 10^{-7}$ & 0.85 & 2.00 & $27 \%$ \\
8 & 0.01 & 0.0001 & 0.15 & 0.22 & 0.12 & 1 & 15.83 & $2.50 \times 10^{-8}$ & 0.17 & 2.09 & $32 \%$ \\
\hline
\end{tabular}

simulate the case where the fracture is embedded in the middle of the isotropic porous medium $\left(\epsilon=0.7, c_{d}=0.001\right)$. The relative error is then $17 \%$ which is slightly higher than the $14 \%$ error, for the corresponding solid wall boundary condition.

\section{Fragmented fractures in isotropic porous media}

Finally, a fragmented fracture is placed in the isotropic structure illustrated in Fig. 5. This simulation is performed applying symmetry boundary condition on lateral walls. For this porous medium of resolution $3000 \times 3000, \epsilon=0.7, c_{d}=0.001$ and fracture size $H=0.4 L$, we have $H / L^{*}=173$, which is a value often found in shale samples as explained in Sect. I. The relative error of the effective permeability computed using the Brinkman model is 1\%. The significance of this configuration is that the fracture does not go through the whole computational domain, thus the porous medium inevitably determines the effective permeability. On the other hand, in the previous examples the preferential path is clearly the channel, therefore, for large fractures the porous medium contribution to the flow and overall permeability of the porous media becomes negligible.

\section{Three-dimensional QSGS}

Recently, Wang et al. ${ }^{27}$ quantified the pore-scale heterogeneity and anisotropy of several shale samples using a geometry-based method, where 3D QSGS structures were generated 


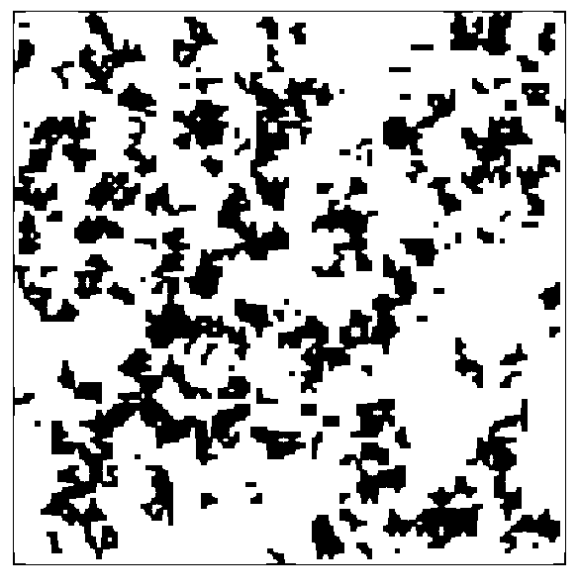

(a)

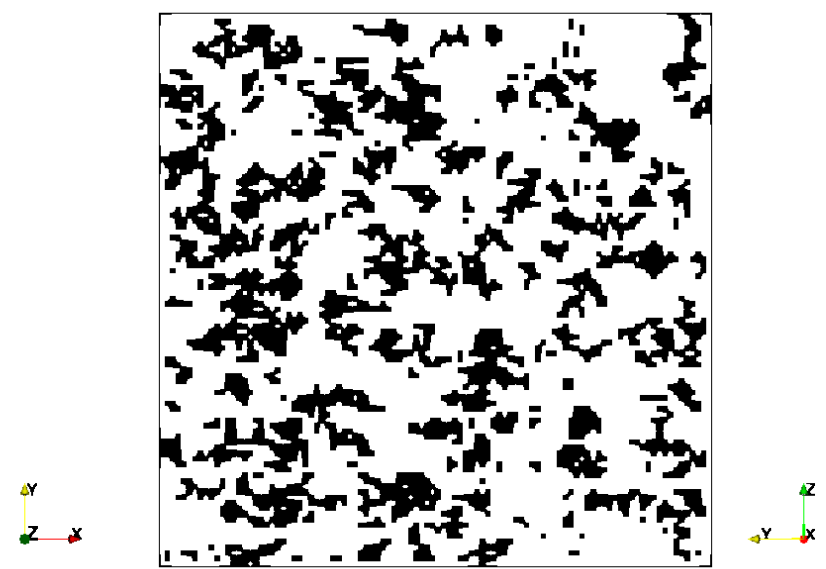

(b)

FIG. 6: Anisotropic 3D QSGS structure (Case 2) where the bedding plane (z) is shown in

(a) and a perpendicular plane $(x)$ in (b). The structure is isotropic in plane $z$ and anisotropic in the other two planes where the pores (shown in black) are elongated.

to have statistical and morphological characteristics close to real samples. The advantage of using this type of reconstruction instead of high-resolution images lies in the fact that we can individually study the impact of the factor of interest, by keeping the rest constant. In our paper, we use similar generating parameters in order to construct random porous media to be realistic representations of the shale matrix, and thus acquire more comprehensive conclusions regarding the accuracy of the Brinkman model for upscaling from the porescale. The full list of parameters of the 3D geometries considered and the corresponding simulation results are summarised in Table III.

\section{Influence of anisotropy}

To start with, we investigate the accuracy of the Brinkman formulation using artificial anisotropic samples with a fracture on top. To this end, we reconstruct two $3 D$ QSGS structures; the first structure is statistically isotropic, while the second is isotropic in the directions parallel to the bed $(x, y)$ and anisotropy appears in the perpendicular direction $(z)$. This type of anisotropy is common in shale, due to the compaction of the rock in the vertical direction. ${ }^{27,34}$

These random porous media are generated using $A R=1$ and 100 (see Cases 1 and 2 
respectively in Table III and Fig. 6 for views of Case 2 geometry). The aspect ratio $(A R)$ herein signifies the ratio of the main directional growth parameters where in the case of transversely isotropic structures this yields $\left(D_{x}=D_{y}\right) / D_{z}$. The flow configuration for the $3 D$ random porous media is identical to the one described in Sect. III A 1. Fixed pressure is applied on the inlet and outlet boundaries, while the rest are treated as stationary walls. The straight fracture $(H=0.02 L)$ is located on top of the porous domain with its height placed on either of the two transverse directions with respect to the pressure gradient.

As previously mentioned, the structures under consideration are either isotropic, or transversely isotropic. Thus, we expect that for the former case the effective permeability results will be practically identical for pressure gradient applied in any of the three directions. Thereby, the same applies for the placement of the fracture. Indeed, our numerical solutions of the Stokes equation show that for $A R=1$ the difference between various pressure gradients and fracture positions is below $0.7 \%$. For the latter case, $\mathrm{d} p / \mathrm{d} x$ and $\mathrm{d} p / \mathrm{d} y$ should give similar $k_{e f f}$ while $\mathrm{d} p / \mathrm{d} z$ is expected to result in inferior values since $k_{z z}<k_{x x} \approx k_{y y}$ for these type of porous media. The fracture position could herein influence the effective permeability. In our simulations, the transversely anisotropic structure $A R=100$ exhibits a difference in effective permeability in the order of $1 \%$ for the two different fracture positions while $\mathrm{d} p / \mathrm{d} x$ is imposed. When the flow direction is driven by pressure gradient in the $z$ direction $(\mathrm{d} p / \mathrm{d} z)$, the resulting $k_{e f f}$ differs by $2 \%$, even though $k_{z z} \approx 0.7 k_{x x}$. This confirms the importance of the fracture flow contribution to the effective permeability. Comparing the two structures, having the same flow configurations, the maximum difference observed in $k_{e f f}$ is in the order of $5 \%$ for $\mathrm{d} p / \mathrm{d} z$, which is expected since the anisotropic structure results in smaller permeability $k_{z z}$. It is worth mentioning that all the aforementioned percentages tend to decrease with increasing $H$.

In the rest of this paper, it may be more meaningful to consider only the setup where a $\mathrm{d} p / \mathrm{d} x$ is applied and the fracture is in the $y$ axis (choosing $\mathrm{d} p / \mathrm{d} y$ and fracture in the $x$ axis would have the same effect). This is due to the fact that is it desirable to choose the fracturing direction parallel to the bedding plane, where the flow is facilitated ${ }^{1}$ and it is the most common flow direction in shale. ${ }^{27}$ The relative error of the Brinkman model for the aforementioned structures is $22 \%$ and $16 \%$ for the isotropic and the transversely isotropic cases respectively (see Case 1 and 2 in Table III). 


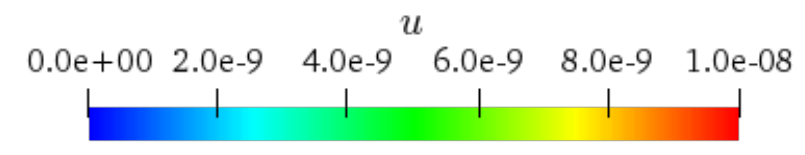

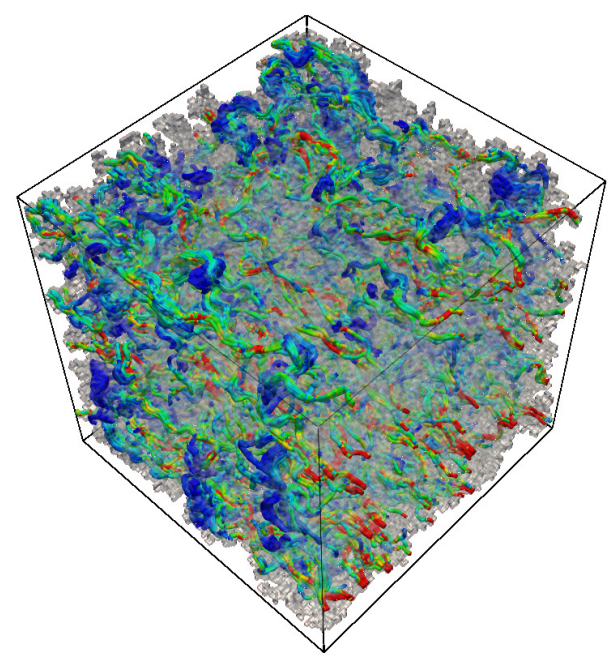

(a)

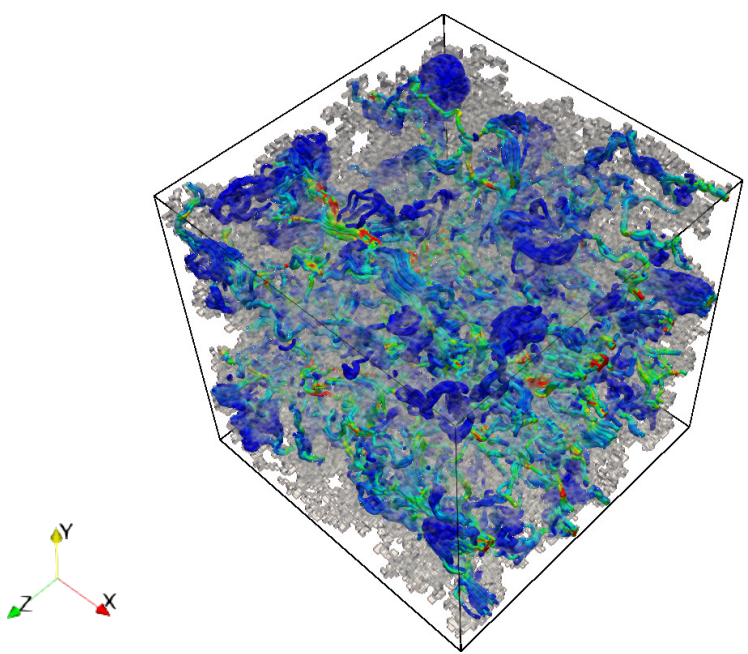

(b)

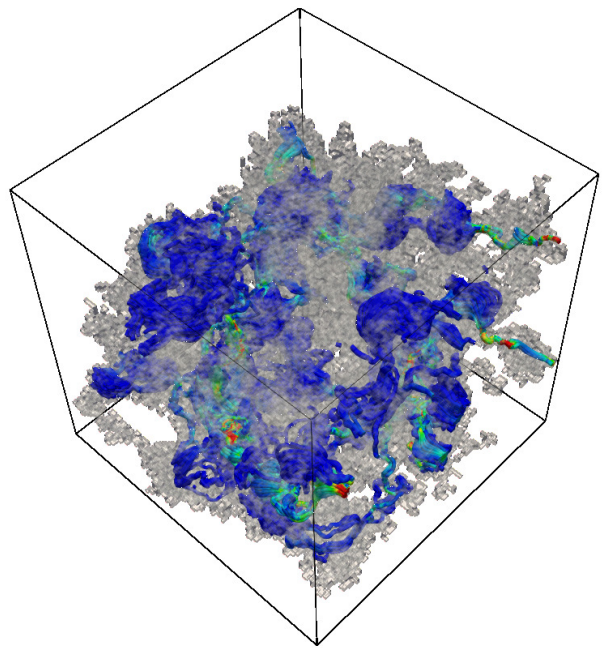

(c)

FIG. 7: The mesh (pore space) of 3D Case 1 (isotropic), Case 7 and Case 8

(heterogeneous) along with velocity streamlines for $\mathrm{d} p / \mathrm{d} x$ shown in (a), (b) and (c) respectively. In the last two subfigures, there are many large pores illustrated with blue colour (due to smaller velocity of the flow there). This is due to the generation process allowing a small portion of the pores to be of greater size $\left(c_{d}^{C}=0.0001\right)$. The grey areas indicate dead end pores, or pore areas with very small velocities.

\section{Influence of heterogeneity}


To study the effect of heterogeneity, several QSGS structures are created with $c_{d}^{R} / c_{d}^{C}=$ 100 and $c_{d}^{R} / c_{d}^{C}=500$, introducing non-uniformity in the pore distribution. It is found that heterogeneity tends to increase with $c_{d}^{R} / c_{d}^{C} \cdot{ }^{27}$ At the same time, transverse isotropy is superimposed to imitate the real porous structure.

The anisotropy factor $r$ used in Table III is defined as

$$
r=\frac{k_{\min }}{\sqrt{k_{\text {int }} k_{\max }}},
$$

where $k_{\min }, k_{i n t}$ and $k_{\max }$ correspond to the minimal, intermediate and maximal component of the diagonal permeability tensor respectively. Values close to unity indicate a statistically isotropic medium, while values close to zero refer to high anisotropy. Particular attention must be paid to the heterogeneous cases, where the matrix proves to be anisotropic even though this is not explicitly determined in the generation process $(A R=1)$. This can be (partly) attributed to the low porosity of the medium in combination with the fact that only a few flow paths are available due to the dominance of the larger pores. When anisotropy is induced by heterogeneity it is not necessary that $k_{z z}$ has the lowest value compared to the rest diagonal terms. On the contrary, this is assured for the anisotropic structures where $A R>1$.

Moreover, it is confirmed that for the same porosity (Cases $4-6$ ), the intrinsic permeability is higher for more heterogeneous structures (Cases 5,6) as the pores have better connectivity. ${ }^{27}$ This can also be justified by the corresponding reduction of tortuosity for the more heterogeneous structures. The porous media of Cases 4 and 8 have the same intrinsic permeability and similar porosities, however they exhibit different relative error of $k_{\text {eff }}(21 \%$ and $32 \%$ respectively). Although they are both constructed based on $c_{d}^{R} / c_{d}^{C}=100$, the proportion of the porosity assigned to each pore size is different $(2 / 1$ versus $1 / 1)$, resulting in Case 8 being more heterogeneous than Case 4 . Additionally, Case 8 is more anisotropic as reflected from the decreased anisotropy factor $r$. In agreement with our conclusions for the 2D porous media presented in Sect. III A 1, the Brinkman estimation for the more heterogeneous and anisotropic structure leads to larger error when the flow is parallel to the bedding plane. On the other hand, comparing Cases 3 and 7 we observe that the significant decrease of $H / L^{*}$ and $r$ for the porous medium flow of Case 7 leads to increase of the Brinkman error as previously detected for $2 D$ structures. Despite the fact that the porous medium of Case 


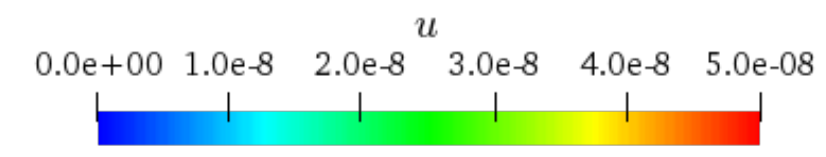

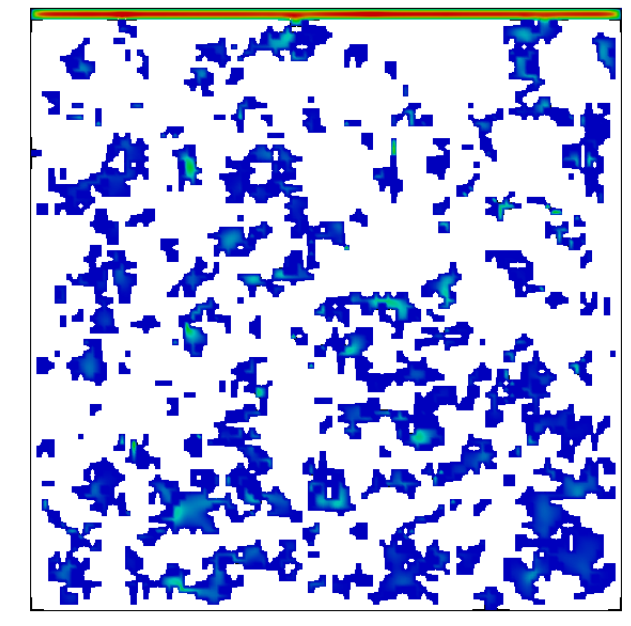

(a)

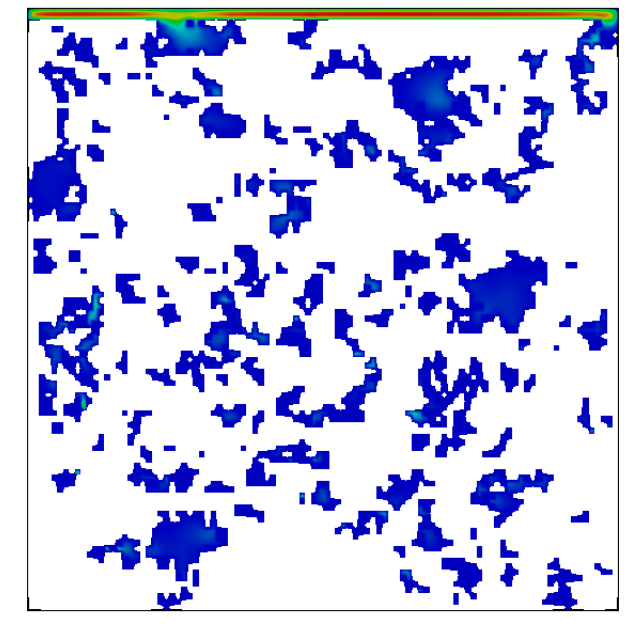

(b)

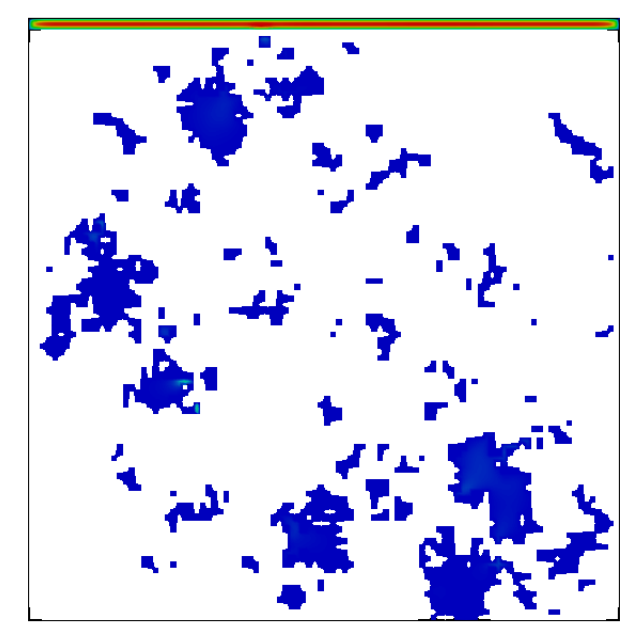

(c)

FIG. 8: Velocity contour plots ( $x$ plane) for the isotropic Case 1 and the heterogeneous Cases 7 and 8 illustrated in (a), (b) and (c) respectively. The fracture size is $H=0.02 L$. The flow is driven by a pressure gradient in the $x$ direction.

7 is less heterogeneous the impact of the other two factors here prevails.

The mesh along with velocity streamlines of a few of the aforementioned porous structures (Cases 1,7 and 8) is depicted in Fig. 7. The focus is on the porous blocks only, thus $H=0$. The heterogeneous cases compared to the isotropic ones have a big proportion of significantly 


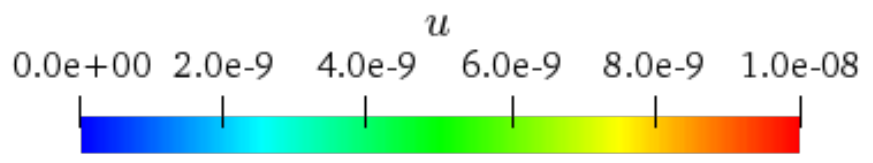

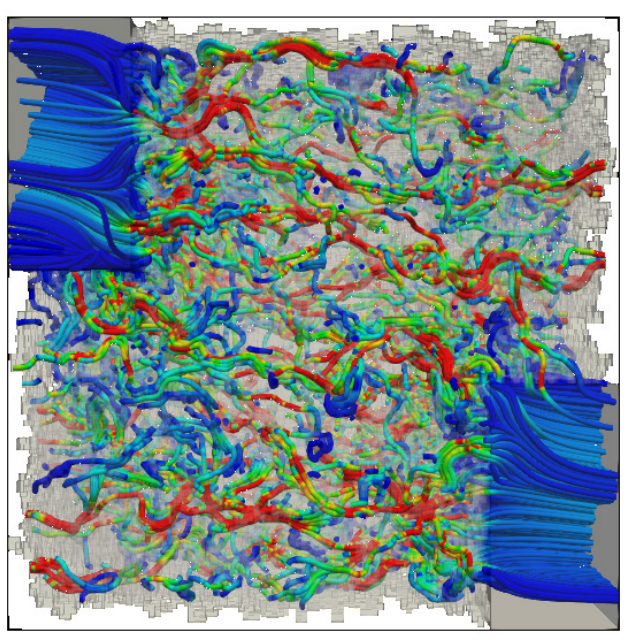

(a)

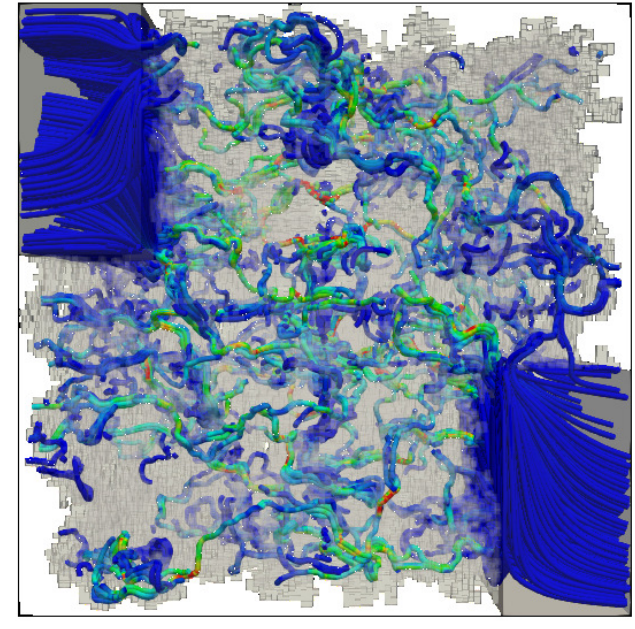

(b)

FIG. 9: Velocity streamlines of the 3D Case 1 (a) and 6 (b), with a fragmented fracture $(H=0.4 L)$ added along the $x$-axis, where $H=91 L^{*}$ for Case 1 and $H=233 L^{*}$ for Case 6 . The Brinkman model underestimates the effective permeability by $2 \%$ and $21 \%$ respectively.

large pores where the flow has low velocity. This can be further observed in Fig. 8 where the velocity contours of same geometries are shown ( $x$ plane). Herein, the porous block is connected to a straight fracture of thickness $H=0.02 L$.

As seen in Fig. 9, a fragmented fracture $(H=0.2 L)$ is placed in the QSGS structures of Case 1 and Case 6, similarly to the 2D configuration illustrated in Fig. 5. The fracture aperture to pore size ratio is about $H / L^{*}=91$ and $H / L^{*}=233$ for the respective geometries, while the corresponding relative error of the Brinkman estimation of effective permeability is $2 \%$ and $21 \%$. Even though the straight fracture configuration gives same error for both structures (see Table III), in this configuration the error differs significantly. This is due to the fact that the permeability tensor is obtained using the initial porous medium, which is now partly covered by the fracture. Since the geometry of Case 1 is more homogeneous, its $\mathbf{k}$ is still representative when we introduce the fracture. However, in the more heterogeneous 
and less permeable geometry of Case 6, the addition of the fracture has a more pronounced impact on the connectivity of the pores, altering the permeability tensor.

If we consider the structures of Table III to have a cell size of $20 \mathrm{~nm}$, then the whole sample size is $L=2 \mu \mathrm{m}$. Thus, the dimensional intrinsic permeabilities of the simulated 3D porous media are in the range of $9.98 \times 10^{-20}$ to $1.70 \times 10^{-18} \mathrm{~m}^{2}$, i.e. approximately 101 to $1722 \mathrm{nD}$. This permeability range, between nano and micro-Darcies, is typical for shale rock. ${ }^{35}$ Additionally, the relevant effective pore size is only a few nanometres $(3-9 \mathrm{~nm})$ and their porosities are low enough for the above structures to be regarded, to some extend, as representative of real shale samples. Consequently, we anticipate that the relative error of the Brinkman model using shale images will be close to the values reported in Table III, i.e. around $20 \%-30 \%$.

\section{B. Upscaling rarefied flows}

Shale gas flow is mostly in the slip and transition regimes, therefore, we examine the performance of Brinkman model, where the intrinsic permeability of porous media is replaced by the apparent permeability due to gas rarefaction effects, on the basic configuration illustrated in Fig. 1, for increasing $K n$. When $K n>0$, the fine-scale results are obtained by solving the linearised BGK equation using the discrete velocity method. ${ }^{4-7}$

The 2D porous medium used herein is isotropic with $\epsilon=0.7$ and $c_{d}=0.001$. Both QSGS grid and mesh have the same resolution $(3000 \times 3000)$. The height of the fracture on top is $H=0.02 L$ and $H=3.5 L^{*}$, where $L^{*}$ is defined in Eq. (8). The fracture size is chosen to be small in order to facilitate the visualisation, since increasing the fracture size the contribution of the porous medium to effective permeability becomes negligible. Additionally, an important factor for this choice is the computational cost of solving the BGK equation which is already demanding for this domain size. Simulations are performed for global $K n=0,0.0001,0.001$ and 0.01, and the velocity contours are shown in Fig. 10 . In order to appropriately understand the rarefied flow behaviour we however use the effective Knudsen number of the average pore and the Knudsen number of the fracture, i.e. $K n^{*}=$ $\lambda / L^{*}$ and $K n^{f}=\lambda / H$, respectively.

It is worthwhile to note that for $K n=0$ the Stokes equation is solved and the respective relative error and graphs are very similar to the ones of $K n=0.0001$; hence they are omitted 


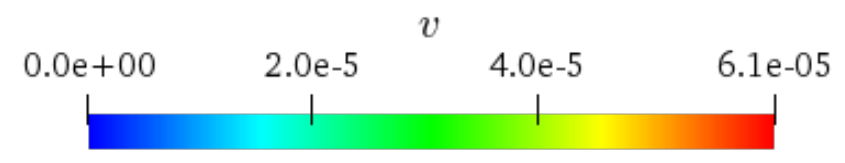

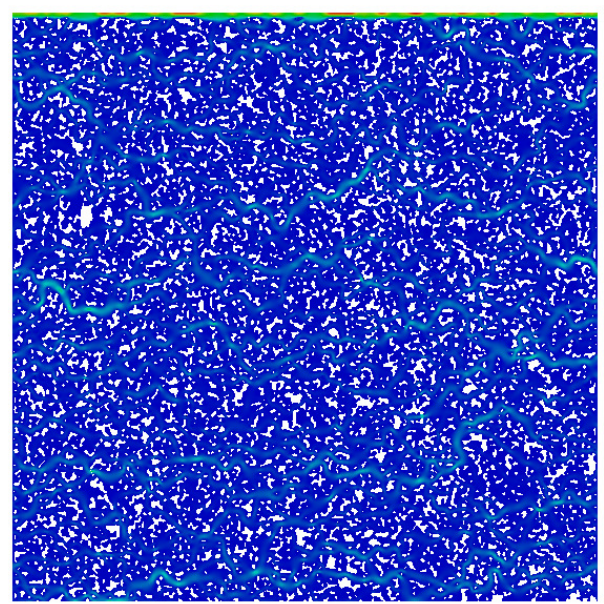

(a) $K n=0.0001$

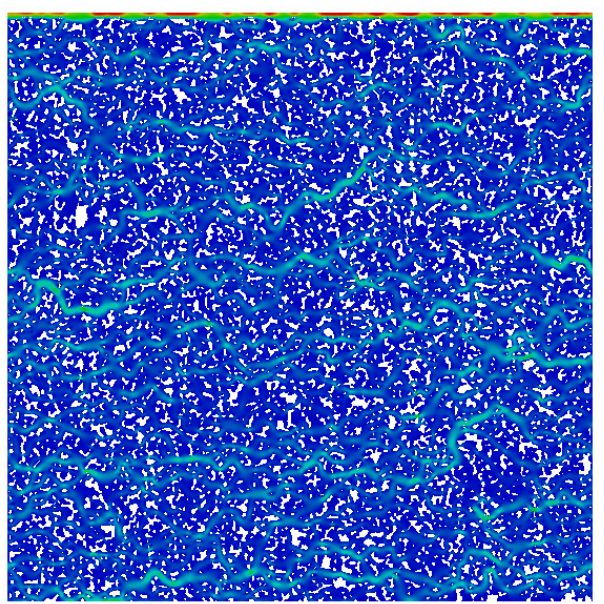

(b) $K n=0.001$

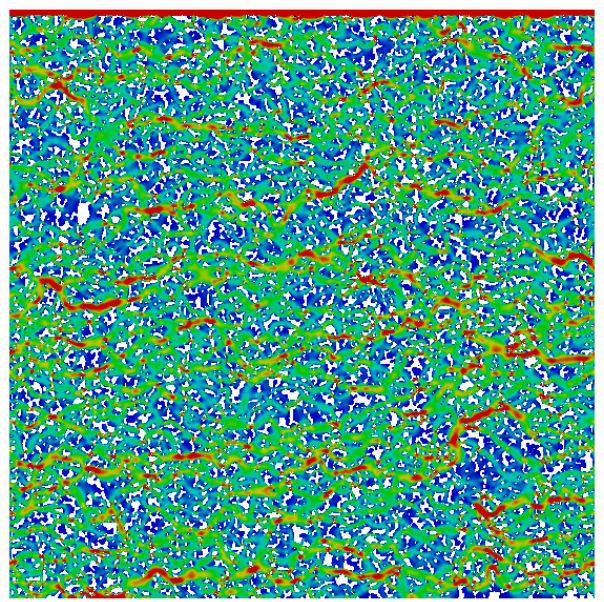

(c) $K n=0.01$

FIG. 10: Contour plots of $v$ for three BGK solutions calculated at (a) $K n=0.0001$, (b)

$K n=0.001$ and (c) $K n=0.01$. The streamwise flow velocity $u$ is normalised by $u_{\text {ref }}=2 \bar{p} L / \mu \sqrt{\pi}$ resulting to $v$. The isotropic porous medium used has $\epsilon=0.7$ and $c_{d}=0.001$ and the straight fracture has an aperture $H=0.02 L$ and $H=3.5 L^{*}$. The flow rate for the largest $K n$ is dramatically increased, indicating significant rarefaction effects.

in Figs. 10 and 11. The full list of results can be found in Table IV. Observing Fig. 10 and the comparative Fig. 11 we can see a dramatic increase in velocity for the case of $K n=0.01$ 


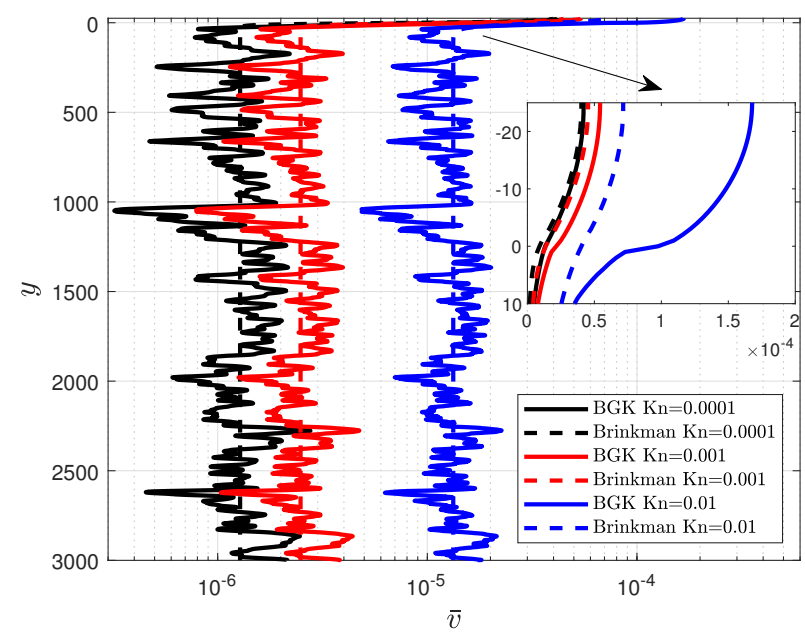

FIG. 11: Streamwise-averaged profiles of $v$, which equals $u$ normalised by $u_{\text {ref }}=2 \bar{p} L / \mu \sqrt{\pi}$. The interface is at $y=0$. The relative error of the Brinkman estimation is $4 \%$ for $K n=0.0001,5 \%$ for $K n=0.001$ and $7 \%$ for $K n=0.01$. Based on the average pore size, $K n^{*}=209 \times K n$ while the effective $K n$ at the fracture is $K n^{f}=60 \times K n$.

compared to $K n=0.001$ and $K n=0.0001$. This indicates that the computed effective $K n$ of the flow $\left(K n^{*}=2\right)$ truly lies in the transition regime.

TABLE IV: The apparent permeability and error of the Brinkman model for rarefied gas flows in 2D QSGS porous medium with a fracture on top with $H=0.02 L$. The column titled $\eta_{K n=0}$ refers to the relative error of the Brinkman estimation when $k_{\text {eff }}^{C}$ is calculated based on the intrinsic permeability of the porous media.

\begin{tabular}{cccccc}
\hline$K n$ & $K n^{*}$ & $K n^{f}$ & $k$ & $\eta$ & $\eta_{K n=0}$ \\
\hline 0 & 0 & 0 & $1.35 \times 10^{-6}$ & $4 \%$ & $4 \%$ \\
0.0001 & 0.02 & 0.006 & $1.45 \times 10^{-6}$ & $4 \%$ & $9 \%$ \\
0.001 & 0.2 & 0.06 & $2.84 \times 10^{-6}$ & $5 \%$ & $50 \%$ \\
0.01 & 2 & 0.6 & $1.52 \times 10^{-5}$ & $7 \%$ & $90 \%$ \\
\hline
\end{tabular}

The coarse-scale results in Fig. 11 indicate that the flow in the porous medium is adequately represented for all three Knudsen numbers tested. We should emphasise here that the permeability tensor used as input for each Brinkman simulation is derived using the BGK results for each corresponding $K n$ number, following the methodology described in Sect. II C.

As far as the area of the straight fracture is concerned, increasing $K n$ the deviation between the BGK and Brinkman results increases. This is due to the fact that in the Brinkman model, practically the Stokes equation is solved at the fracture, which are proven 
to break down in the transition and free flow regimes as mentioned in Sect. IID. Namely, for $K n=0.01$ the flow in the fracture is significantly underestimated, therefore, the relative error of effective permeability increases to $7 \%$. Nevertheless, this error is still small thanks to the precise $\mathbf{k}$ used, which lays a firm foundation for upscaling. This is of central importance, since using $\mathbf{k}$ obtained from the Stokes equation instead of the BGK model has a notable effect on the coarse-scale effective permeability. For example, it results in underestimation of permeability up to $90 \%$ at $K n=0.01$.

Due to the small fracture size, its contribution to the total mass flow is limited hence the error involved in the value of $k_{\text {eff }}^{C}$ is not so significant. On the other hand, in the case of a larger fracture for the same global $K n, K n^{f}$ decreases, resulting to the Brinkman model being accurate for a larger range of global $K n$. Consequently, the relative error is expected to be even smaller.

\section{CONCLUSIONS}

In this paper, we have thoroughly investigated the error of the effective permeability estimation when the Brinkman model is used to upscale shale permeability from the porescale. We performed 2D and 3D flow simulations using random porous media generated with the QSGS method where several morphological properties can be controlled. Extensive analysis has been performed for both continuum and rarefied gas flows which are often encountered in shale. To the best of our knowledge, this is the first time that the accuracy of the Brinkman model using realistic porous media and flow conditions is examined.

For continuum flows, a parametric study of isotropic 2D structures reveals that homogeneity favours the estimation of the Brinkman model, no matter whether homogeneity is resulted from high porosity or small particle size. Additionally, anisotropy in 2D geometries, when the flow is perpendicular to bed, leads to reduced error. In other words, for highly tortuous porous media, the increase of the $H / L^{*}$ factor is associated with more accurate prediction of the effective permeability. However, when the flow is parallel to the bedding plane, this error tends to increase. Our simulations using artificial 3D random porous media demonstrate that a reasonable relative error in the estimation of effective permeability occurs when the Brinkman formulation is used. These 3D porous media are carefully constructed to resemble the properties of real shale sample images of high resolution. Even 
though the cases studied are quite complex and the impact of each parameter cannot be easily uncoupled, the same conclusions as in 2D apply. The Brinkman model is less reliable when used for porous media that exhibit high heterogeneity and anisotropy. Most of the flow configurations performed have a straight fracture on top of the porous region as it is common in the literature. However, a fragmented channel is also placed in two different porous media to demonstrate a more realistic example of a shale sample with a microfracture. The Brinkman-derived coarse-scale permeability of both configurations is in good agreement with the fine-scale model.

The suitability of the Brinkman model is also verified, for the first time, on rarefied gas flows where the permeability of porous media is chosen as the apparent permeability which is a function of gas pressure. Microscopic 2D simulations spanning a wide range of Knudsen numbers are performed by solving the linearised BGK equation. The apparent permeability tensor is then utilised as input for the macroscopic representation of Brinkman leading to well predicted effective permeability for rarefied gas flows. Neglecting the rarefaction effects is proven to result in significant underestimation of the macroscopic property.

We must remark that in this work, $\mu_{e f f}=\mu$ is adopted in the Brinkman equation and the accuracy of the results is adequate for the realistic cases simulated. Thus, we recommend to avoid using complex and controversial alternatives suggested in the literature when the aim is to predict flow properties of shale.

In conclusion, according to our numerous 2D and 3D simulation results the effective permeability extracted from the Brinkman formulation compares favourably to its fine-scale counterpart computed by solving the Stokes and the Boltzmann model equations. We thus have confidence that the use of Brinkman model is suitable for shale permeability upscaling from the pore-scale.

\section{ACKNOWLEDGMENTS}

This work is financially supported by the Engineering and Physical Sciences Research Council (EPSRC) in the UK under Grant No. EP/R041938/1. Results were obtained using the EPSRC funded ARCHIE-WeSt High Performance Computer (EPSRC Grant No. $\mathrm{EP} / \mathrm{K} 000586 / 1)$. 


\section{DATA AVAILABILITY}

The data that support the findings of this study are available from the corresponding author upon reasonable request.

\section{REFERENCES}

${ }^{1}$ L. Ma, K. G. Taylor, P. D. Lee, K. J. Dobson, P. J. Dowey, and L. Courtois, "Novel 3D centimetre-to nano-scale quantification of an organic-rich mudstone: The Carboniferous Bowland Shale, Northern England," Mar. Pet. Geol. 72, 193-205 (2016).

${ }^{2} \mathrm{~T}$. Wu, X. Li, J. Zhao, and D. Zhang, "Multiscale pore structure and its effect on gas transport in organic-rich shale," Water Resour. Res. 53, 5438-5450 (2017).

${ }^{3}$ X. Wang and J. Sheng, "Gas sorption and non-Darcy flow in shale reservoirs," Pet. Sci. 14, 746-754 (2017).

${ }^{4}$ L. Wu, M. T. Ho, L. Germanou, X.-J. Gu, C. Liu, K. Xu, and Y. Zhang, "On the apparent permeability of porous media in rarefied gas flows," J. Fluid Mech. 822, 398-417 (2017).

${ }^{5}$ L. Germanou, M. T. Ho, Y. Zhang, and L. Wu, "Intrinsic and apparent gas permeability of heterogeneous and anisotropic ultra-tight porous media," J. Nat. Gas Sci. Eng. 60, 271-283 (2018).

${ }^{6}$ M. T. Ho, L. Zhu, L. Wu, P. Wang, Z. Guo, Z.-H. Li, and Y. Zhang, "A multi-level parallel solver for rarefied gas flows in porous media," Comput. Phys. Commun. 234, 14-25 (2019).

${ }^{7}$ M. T. Ho, L. Zhu, L. Wu, P. Wang, Z. Guo, J. Ma, and Y. Zhang, "Pore-scale simulations of rarefied gas flows in ultra-tight porous media," Fuel 249, 341-351 (2019).

${ }^{8}$ J. F. Gale, S. E. Laubach, J. E. Olson, P. Eichhuble, and A. Fall, "Natural Fractures in shale: A review and new observations," Am. Assoc. Pet. Geol. Bull. 98, 2165-2216 (2014).

${ }^{9}$ M. H. Anders, S. E. Laubach, and C. H. Scholz, "Microfractures: A review," J. Struct. Geol. 69, 377-394 (2014).

${ }^{10} \mathrm{H}$. C. Brinkman, "A calculation of the viscous force exerted by a flowing fluid on a dense swarm of particles," Appl. Sci. Res. 1, 27 (1949).

${ }^{11}$ C. Soulaine, F. Gjetvaj, C. Garing, S. Roman, A. Russian, P. Gouze, and H. A. Tchelepi, "The Impact of Sub-Resolution Porosity of X-ray Microtomography Images on the Permeability," Transp. Porous Media 113, 227-243 (2016). 
${ }^{12}$ L. M. Keller and L. Holzer, "Image-Based Upscaling of Permeability in Opalinus Clay," J. Geophys. Res. Solid Earth 123, 285-295 (2018).

${ }^{13}$ P. Popov, G. Qin, L. Bi, Y. Efendiev, R. E. Ewing, and J. Li, "Multiphysics and Multiscale Methods for Modeling Fluid Flow Through Naturally Fractured Carbonate Karst Reservoirs," SPE Reserv. Eval. Eng. 12, 218-231 (2009).

${ }^{14}$ M. Krotkiewski, I. S. Ligaarden, K.-A. Lie, and D. W. Schmid, "On the Importance of the Stokes-Brinkman Equations for Computing Effective Permeability in Karst Reservoirs," Commun. Comput. Phys. 10, 1315-1332 (2011).

${ }^{15}$ G. S. Beavers and D. D. Joseph, "Boundary conditions at a naturally permeable wall," J. Fluid Mech. 30, 197-207 (1967).

${ }^{16}$ A. Terzis, I. Zarikos, K. Weishaupt, G. Yang, X. Chu, R. Helmig, and B. Weigand, "Microscopic velocity field measurements inside a regular porous medium adjacent to a low Reynolds number channel flow," Phys. Fluids 31, 042001 (2019).

${ }^{17}$ G. Karniadakis, A. Beskok, and N. Aluru, Microflows and Nanoflows: Fundamentals and Simulation (Springer, 2005).

${ }^{18}$ J. L. Auriault, "On the domain of validity of Brinkman's equation," Transp. Porous Media 79, 215-223 (2009).

${ }^{19}$ D. A. Nield and A. Bejan, Convection in Porous Media (Springer, 2013).

${ }^{20}$ N. Martys, D. P. Bentz, and E. J. Garboczi, "Computer simulation study of the effective viscosity in Brinkman's equation," Phys. Fluids 6, 1434-1439 (1994).

${ }^{21}$ J. A. Ochoa-Tapia and S. Whitaker, "Momentum transfer at the boundary between a porous medium and a homogenous fluid-I. Theoretical development," Int. J. Mass Transf. 38, 2635-2646 (1995).

${ }^{22}$ F. J. Valdes-Parada, J. Alberto Ochoa-Tapia, and J. Alvarez-Ramirez, "On the effective viscosity for the Darcy-Brinkman equation," Physica A 385, 69-79 (2007).

${ }^{23}$ M. Sahraoui and M. Kaviany, "Slip and no-slip velocity boundary conditions at interface of porous, plain media," Int. J. Heat Mass Transf. 35, 927-943 (1992).

${ }^{24}$ M. Wang, J. Wang, N. Pan, and S. Chen, "Mesoscopic predictions of the effective thermal conductivity for microscale random porous media," Phys. Rev. E 75, 1-10 (2007).

${ }^{25}$ L. Chen, W. Fang, Q. Kang, J. De'Haven Hyman, H. S. Viswanathan, and W.-Q. Tao, "Generalized lattice Boltzmann model for flow through tight porous media with Klinkenberg's effect," Phys. Rev. E 91, 033004 (2015). 
${ }^{26}$ L. Chen, Q. Kang, Z. Dai, H. S. Viswanathan, and W. Tao, "Permeability prediction of shale matrix reconstructed using the elementary building block model," Fuel 160, 346-356 (2015).

${ }^{27}$ Z. Wang, X. Jin, X. Wang, L. Sun, and M. Wang, "Pore-scale geometry effects on gas permeability in shale," J. Nat. Gas Sci. Eng. 34, 948-957 (2016).

${ }^{28}$ J. Wang, Q. Kang, Y. Wang, R. Pawar, and S. S. Rahman, "Simulation of gas flow in micro-porous media with the regularized lattice Boltzmann method," Fuel 205, 232-246 (2017).

${ }^{29}$ R. Guibert, M. Nazarova, P. Horgue, G. Hamon, P. Creux, and G. Debenest, "Computational Permeability Determination from Pore-Scale Imaging: Sample Size, Mesh and Method Sensitivities," Transp. Porous Media 107, 641-656 (2015).

${ }^{30}$ R. Guibert, P. Horgue, G. Debenest, and M. Quintard, "A Comparison of Various Methods for the Numerical Evaluation of Porous Media Permeability Tensors from Pore-Scale Geometry," Math. Geosci. 48, 329-347 (2016).

${ }^{31}$ L. J. Klinkenberg, "The Permeability Of Porous Media To Liquids And Gases," Am. Pet. Inst. , 200-213 (1941).

${ }^{32} \mathrm{Q}$. Zhang and A. Prosperetti, "Pressure-driven flow in a two-dimensional channel with porous walls," J. Fluid Mech. 631, 1-21 (2009).

${ }^{33}$ A. Duda, Z. Koza, and M. Matyka, "Hydraulic tortuosity in arbitrary porous media flow," Phys. Rev. E 84, 036319 (2011).

${ }^{34} \mathrm{M}$. Mokhtari and A. N. Tutuncu, "Characterization of anisotropy in the permeability of organic-rich shales," J. Pet. Sci. Eng. 133, 496-506 (2015).

${ }^{35}$ N. Chakraborty, Z. Karpyn, S. Liu, and H. Yoon, "Permeability evolution of shale during spontaneous imbibition," J. Nat. Gas Sci. Eng. 38, 590-596 (2017). 